\title{
A single sunburn influences the immunological component of human skin for up to 14 days
}

Suzanne M. Pilkington', Norah Al-Gazaaq²,Sharon Murphy², Anna Nicolaou² and Lesley E. Rhodes ${ }^{1}$ ${ }^{1}$ Centre for Dermatology, School of Biological Sciences, and ${ }^{2}$ School of Health Sciences, Faculty of Biology Medicine and Health, University of Manchester, Manchester, UK.

\section{INTRODUCTION}

Sunburn is prevalent, and while the clinically apparen effects disappear relatively quickly, repeated episodes have longer term consequences including skin cancer. Proinflammatory eicosanoid expression and leukocytic infiltration characterise the peak of the sunburn response ${ }^{1}$ however, little is known of these processes during the resolution phase.

Therefore, in human skin, we examined the changes in eicosanoid expression and leukocytic infiltration up to 14 days post acute sunburn.

\section{METHODS}

- Ethical approval was granted by the North Manchester NHS Research Ethics Committee (ref 11/NW/0567).

- Exclusion criteria were sunbed use and recent sunbathing, pre-existing skin conditions including skin cancer, use of anti-inflammatory medication and omega-3 supplements.

- 13 healthy white Caucasian adults were recruited (2058 years, $5 \mathrm{M} / 8 \mathrm{~F}$, phototype I-III)

- Photoprotected buttock skin was exposed to a single 3x MED of broadband ultraviolet radiation (UVR; Waldman UV6; 280-400nm) at multiple sites and changes in skin erythema were measured over a 14 day period using a spectrophotometer (CM600d, Konica Minolta Sensing Europe).

- Biopsies and suction blister fluid were sampled from unexposed and UVR-exposed skin across the 14 day time-course and analysed by immunohistochemistry (neutrophil elastase, CD4, CD8, CD68, CD1a, COX-2, EP4) and LC-MS/MS (eicosanoids).

\section{RESULTS}

1) The erythemal response to UVR is not fully resolved at day 14 post-exposure

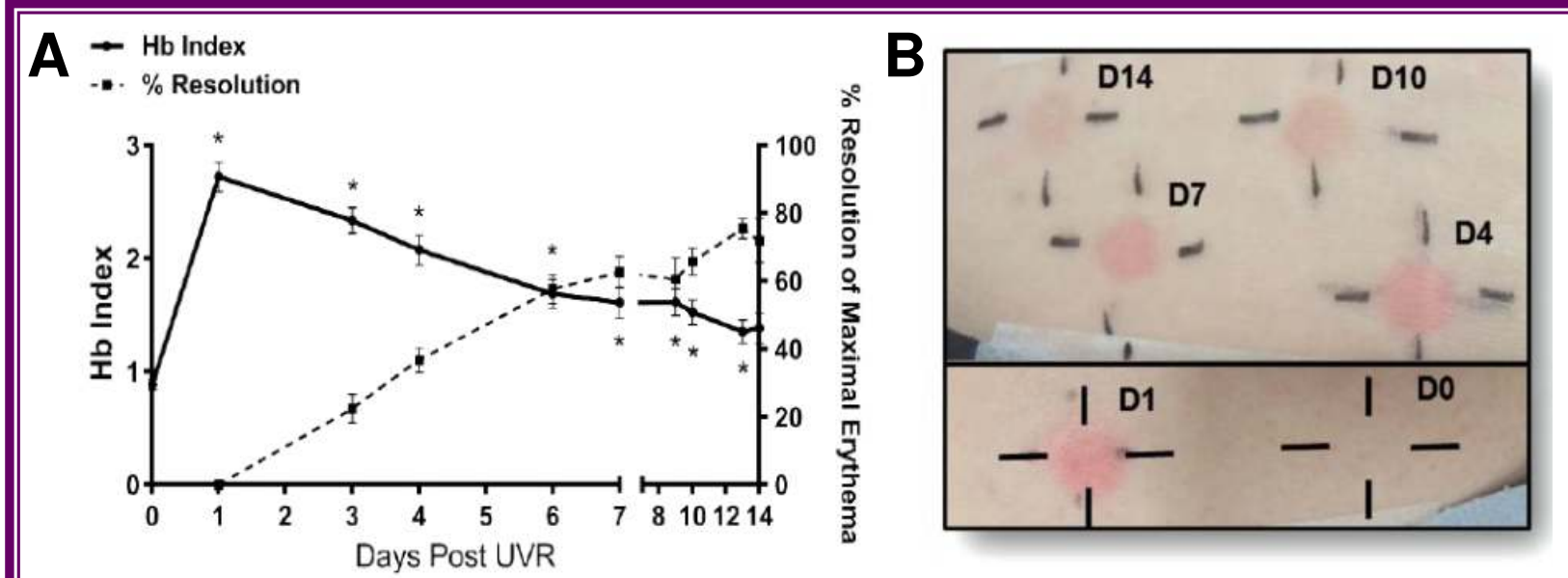

A) Hemoglobin $(\mathrm{Hb})$ Index and \% resolution of eythema. By day 7 postUVR, eythema had resolved by $60 \%$ and by day 14 had $80 \%$ resolved, $p<0.05$ compared to unexposed skin. B) Photograph of skin showing the sunburn response to 3xMED of UVR over the 14 day resolution period.

2) Epidermal expression of COX-2 peaks at day 1 while $\mathrm{PGE}_{2}$ receptor EP4 is elevated up to day 10

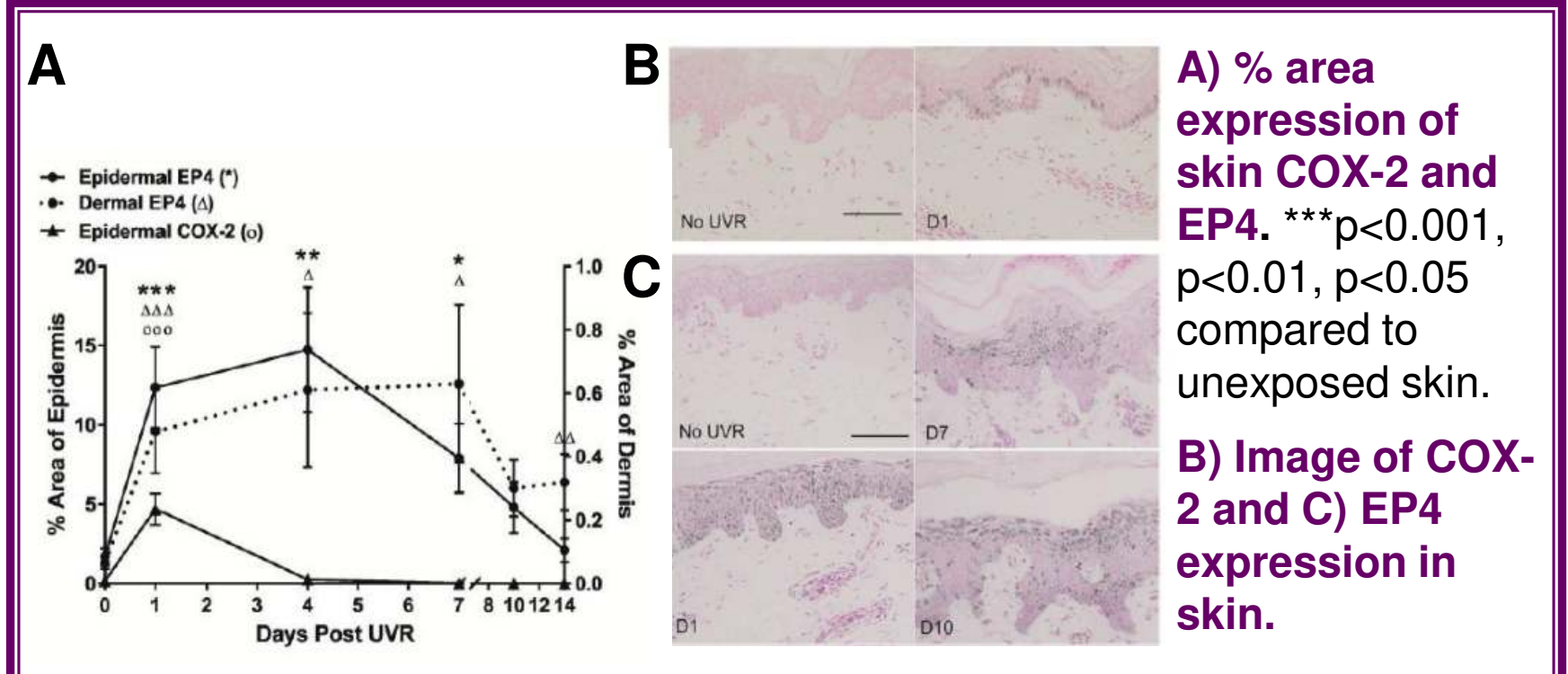

\section{RESULTS}

3) Epidermal pro-inflammatory prostaglandin and hydroxy fatty acid levels peak at day 1 post UVR

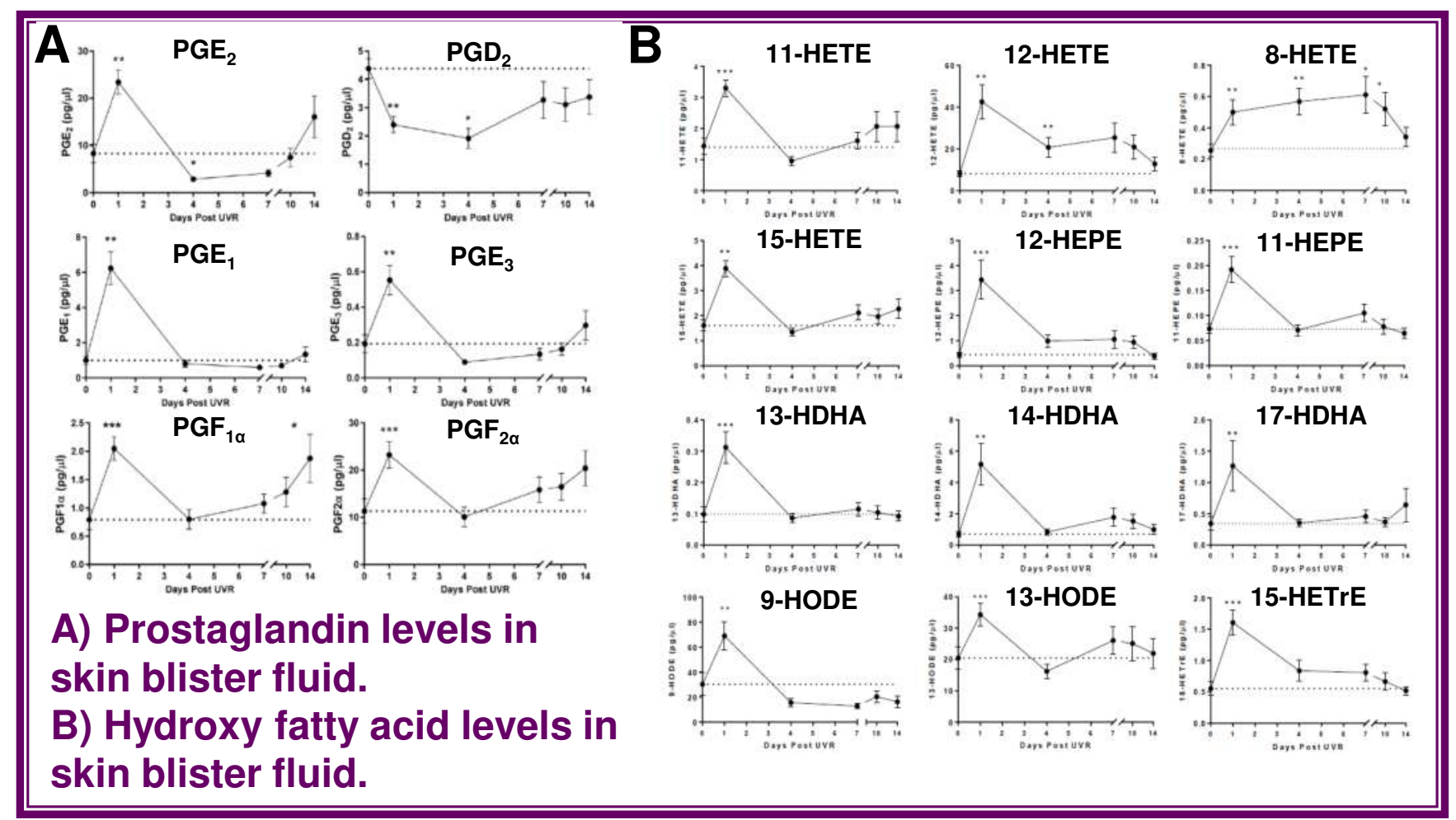

4) Macrophage, CD4+ and CD8+ T cell numbers remain elevated up to 14 days and Langerhans cell numbers are depleted for up to 10 days, post-UVR exposure

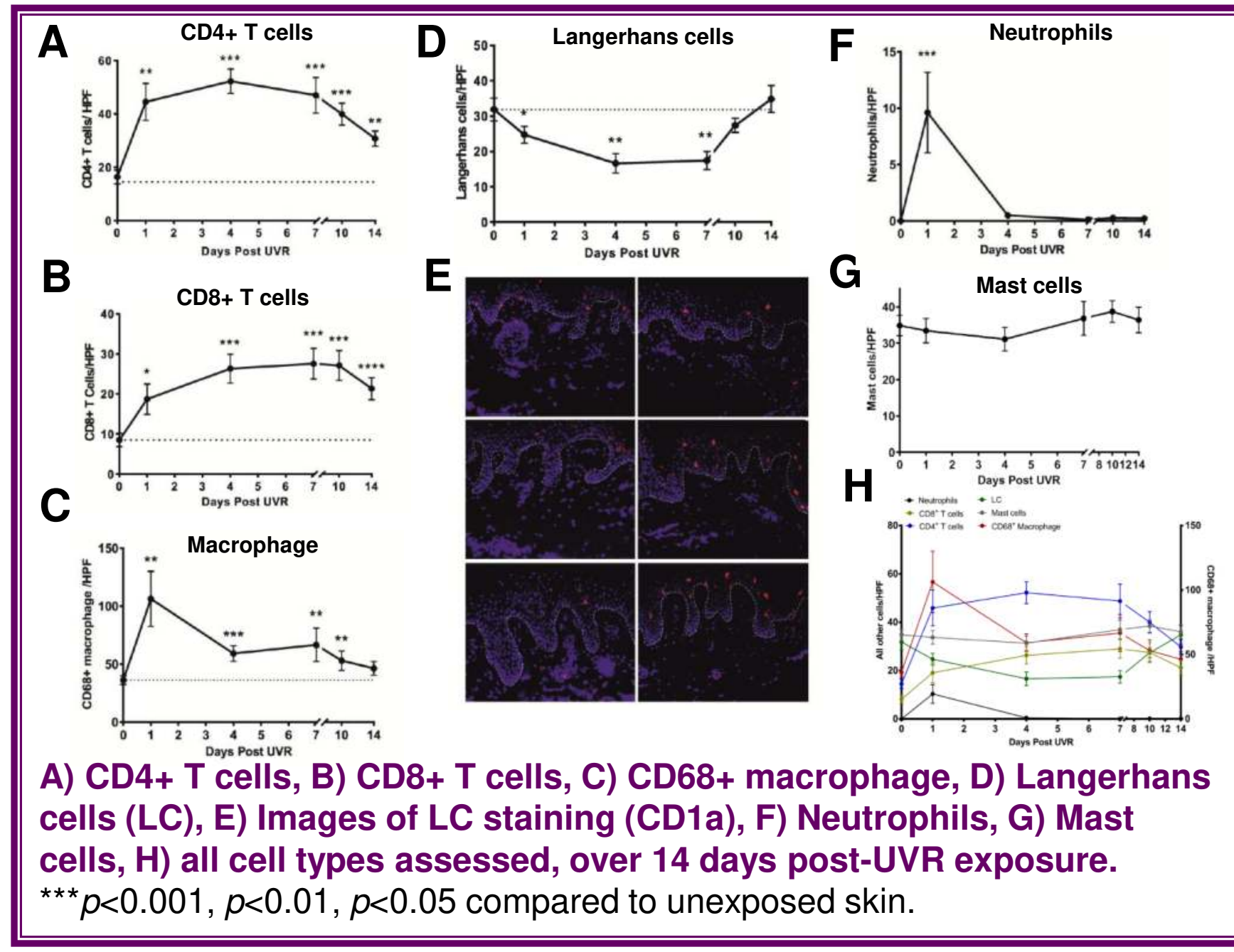

\section{CONCLUSIONS}

- A single exposure to high dose UVR results in a sunburn response that lasts 10 to 14 days in the skin of individuals with phototype I-III.

- This is characterised by ongoing erythema and elevated expression of prostaglandin receptor EP4, which could facilitate maintenance of the erythemal response.

- An inflammatory infiltrate of innate and adaptive immune cells is also sustained up to 14 days, while LC are depleted for 10 days, potentially leaving the skin immunocompromised during this time.

- Further study of the sunburn resolution phase could elucidate the contribution of these inflammatory/immune changes to skin cancer.

\section{REFERENCES}

1) Rhodes LE et al. The sunburn response in human skin is characterized by sequential eicosanoid profiles that may mediate its early and late phases (2009) FASEB J 23 (11):3947 